\title{
The nasal symbiont Staphylococcus epidermidis shapes the cellular environment to decrease expression of SARS-CoV-2 entry factors in nasal epithelium
}

Jeong Yeon Ji

Seoul National University College of Medicine

Ara Jo

Seoul National University College of Medicine

Jina Won

Seoul National University College of Medicine

Chan Hee Gil

Seoul National University College of Medicine

Haeun Shin

Seoul National University College of Medicine

Sujin Kim

Seoul National University College of Medicine

Yung Jin Jeon

Busan Gyeongsang Daehakgyo: Busan Kyungsang College

Hyun Jik Kim ( $\square$ hyunjerry@snu.ac.kr)

Seoul National University College of Medicine https://orcid.org/0000-0001-8631-928X

Short report

Keywords:

Posted Date: February 18th, 2021

DOl: https://doi.org/10.21203/rs.3.rs-208802/v1

License: (c) (1) This work is licensed under a Creative Commons Attribution 4.0 International License. Read Full License 


\section{Abstract \\ Background}

Emerging evidence indicates that severe acute respiratory syndrome-related coronavirus-2 (SARS-CoV-2) targets the human nasal epithelium via the principal entry factors angiotensin-converting enzyme 2 (ACE2) and transmembrane serine protease 2 (TMPRSS2), which are highly expressed in the nasal epithelium. However, little is known about suppressive biologics against SARS-CoV-2 entry factors. Here, we report that the nasal commensal Staphylococcus epidermidis altered the host transcriptional response against SARS-CoV-2 in the nasal epithelium by reducing ACE2 and TMPRSS2 gene expression in concert with an increase in serine-peptidase inhibitors.

\section{Results}

Our data reveal that $A C E 2$ was more abundantly expressed in nasal epithelial (NHNE) cells than bronchial epithelial cells, and inoculation with S. epidermidis reduced ACE2 transcription in NHNE cells. Our data also show that TMPRSS2 mRNA was significantly decreased in NHNE cells and that $S$. epidermidis colony number in human nasal mucus was inversely correlated with ACE2 and TMPRSS2 gene expression in the nasal mucosa. In addition, levels of the serine-peptidase inhibitors SERPINE1 and SERPINE2 were significantly increased by $S$. epidermidis, and this accompanied reduction of TMPRSS2 transcription in nasal epithelial cells.

\section{Conclusion}

These results characterize the $S$. epidermidis-regulated host transcriptional response restricting SARSCoV-2 entry to the nasal epithelium via downregulation of receptors and host protease for SARS-CoV-2 cellular invasion coupled with SERPINE1 and SERPINE2 induction.

\section{Background}

At present, the world is suffering from a pandemic infection of severe acute respiratory syndrome-related coronavirus-2 (SARS-CoV-2), which causes coronavirus disease 2019 (COVID-19) and leads to acute respiratory distress syndrome or viral pneumonia with severe damage to the lungs $[1,2]$. Currently, research on development of vaccines against SARS-CoV-2 is ongoing worldwide, and interest in effective SARS-CoV-2 therapeutics is increasing rapidly $[3,4]$. To succeed in development of a therapeutic or vaccine against SARS-CoV-2, knowledge of the exact target cells where SARS-CoV-2 enter the host and the mechanism of infection in the respiratory tract is essential.

Many respiratory virus families require maturation cleavage of viral surface glycoproteins after binding to a specific receptor, generally realized by host serine proteases. The cellular infectivity of a respiratory 
virus to the respiratory epithelium depends on the distribution of receptors and activity of host serine proteases in the respiratory tract [5-7]. It is becoming increasingly apparent that nasal epithelial cells are the primary target of SARS-CoV-2, and the nasal epithelium is regarded as a portal for initial infection and/or transmission of SARS-CoV-2 to the respiratory tract [8, 9]. SARS-CoV-2 employs angiotensin converting enzyme 2 (ACE2) as a receptor for internalization, and the binding affinity of the spike (S) protein of SARS-CoV-2 to ACE2 was found to be a major determinant of SARS-CoV-2 nasal epithelium cellular infection $[10,11]$. Host proteases are involved in cellular invasion by SARS-CoV-2, and transmembrane serine protease 2 (TMPRSS2) is indicated as the principal host protease to mediate cleavage of SARS-CoV-2 S protein in nasal epithelial cells [8]. In this regard, it is of immediate interest to determine whether localized suppression of ACE2 in the nasal epithelium restricts cellular invasion of SARS-CoV-2 and inhibits viral replication in the respiratory tract. Likewise, suppression of TMPRSS2driven SARS-CoV-2 S protein activation might provide a new therapeutic approach to prevent SARS-CoV2-caused respiratory infection.

The respiratory microbiome is constantly exposed to inhaled pathogens and is known to influence the course of host immune responses. Inhaled pathogens including respiratory viruses encounter the host immune system through the nasal passage, and the microbial characteristics of the nasal mucus directly impact initiation of the innate immune response $[12,13]$. Thus, insights into the human nasal mucus microbiome can provide fundamental information regarding defense mechanisms against respiratory virus infection, and understanding of microbiome-regulated immune factors contributes to discovery of new concepts of viral infection control [14].

Our previous study identified Staphylococcus epidermidis as the most abundant constituent in human nasal mucus and showed that $S$. epidermidis accelerated clearance of influenza virus from the nasal epithelium through interferon-related immune responses [13]. Here, we investigated if $S$. epidermidis plays a role in reinforcing the antiviral innate immune response at the nasal epithelium to determine any contribution to suppressing SARS-CoV-2 infection that targets nasal epithelial cell as onset of infection. We investigated the correlation between $S$. epidermidis and SARS-CoV-2 entry factors, which are mainly distributed on nasal epithelial cells, and found that the human nasal commensal $S$. epidermidis impeded entry of SARS-CoV-2 into nasal epithelial cells by reducing the expression of ACE2 and TMPRSS2.

Mechanistically, we show that $S$. epidermidis protects the nasal epithelium from spread of SARS-CoV-2 by enhancing the activity of serine protease inhibitors. The current findings provide evidence of nasal microbiome-altered cellular environments associated with disturbance of SARS-CoV-2 entry factors in the human nasal epithelium.

\section{Results}

In particular, ACE2 has been detected in both nasal and bronchial epithelium, and ACE2 gene expression has been recently reported largely in nasal epithelial cells including secretory cells and ciliated cells, which are central to SARS-CoV-2 pathogenesis in the upper airway [8]. We evaluated ACE2 RNA expression in human nasal mucosa $(\mathrm{N}=4)$ and lung tissue $(\mathrm{N}=4)$ and compared it to that of DPPIV, which 
encodes a known viral receptor for MERS-CoV, and ST6GAL 1 and ST3GAL4, which are important for synthesis of $a(2,6)$-linked and $a(2,3)$-linked sialic acids recognized by influenza virus $[15,16]$. Real-time PCR revealed that mRNA expression of DPPIV in lung parenchymal tissue was significantly higher than in the nasal mucosa, and neither mRNA expression of ST6GAL 1 or ST3GAL4 was significantly different in human nasal mucosa and lung tissue. Unlike the expression of other viral receptors, the mean level of ACE2 mRNA was higher in the human nasal mucosa $\left(1.7 \times 10^{9}\right)$ than in lung tissue $\left(4.8 \times 10^{7}\right)$ (Fig. $\left.1 \mathrm{a}\right)$. Immunohistochemistry (IHC) for ACE2 protein was performed using human nasal mucosa of middle turbinate to determine whether ACE2 protein is mainly present in nasal mucosa (Additional file 1: Figure S1). Although the expression of ACE2 protein was observed in a part of submucous gland, IHC results showed that positive DAB (3,3'-diaminobenzidine) staining of ACE2 protein was highly increased in the nasal epithelium relative to the subepithelial area of the human nasal mucosa (Fig. 1b).

To further characterize the expression of viral receptors in nasal epithelial cells, we examined ACE2, DPPIV, ST6GAL 1, and ST3GAL4 expression within air-liquid interface cultures of normal human nasal epithelial (NHNE) cells, and the results were compared to those of normal human bronchial epithelial (NHBE) cells. Based on results using this in vitro system, we confirmed increased ACE2 mRNA expression in nasal epithelial cells, and mRNA level of ACE2 was higher in NHNE than NHBE cells (Fig. 1c). We found that DPPIV expression was higher in NHBE cells, and no significantly different expression of ST6GAL 1 and ST3GAL4 was observed between NHNE and NHBE cells. To clarify the cell subsets targeted by SARSCoV-2 in the human nasal epithelium, we investigated gene expression of ACE2 depending on nasal epithelial cell subset through single-cell RNA sequencing (scRNA-seq). We confirmed increased normalized ACE2 expression in both suprabasal cells and secretory-like NHNE cells (Fig. 1d). These data suggest that the nasal epithelium is the primary target of SARS-CoV-2 transmission, and SARS-CoV-2 infection is spread to the respiratory tract after intracellular entry via nasal epithelial cells.

To determine the correlation between the abundant nasal commensal S. epidermidis and entry factors of SARS-CoV-2 in the nasal epithelium, NHNE cells from five healthy subjects were inoculated with $S$. epidermidis isolated from healthy human nasal mucus, for $24 \mathrm{~h}$ at a multiplicity of infection (MOI) of 0.25 (Additional file 1: Figure S2). We performed Gene Ontology (GO) enrichment analysis of scRNA-seq data using cell lysates from the $S$. epidermidis-inoculated NHNE cells to confirm the effect of $S$. epidermidis in restricting host entry factors of SARS-CoV-2. The terms associated with virus receptor activity were analyzed, and top significant terms included "entry into host cells," "entry into other organism involved in symbiotic interaction," "viral entry into host," and "viral life cycle" (Fig. 2a). The terms associated with serine-type peptidase activity were also examined, and the results revealed top significant terms of "virus receptor activity," "serine-type endopeptidase inhibitor activity," "serine-type peptidase activity," "peptidase activity," and "receptor binding" (Fig. 2b). 
We used scRNA-seq to characterize the response of the nasal epithelium to $S$. epidermidis inoculation. Significant gene populations (fold change $\geq$ or $<1.5$ and normalized data $(\log 2) \geq$ or $<2.0$ ) in $S$. epidermidis-inoculated NHNE cells were compared to those from NHNE cells without S. epidermidis inoculation. The scatter plot data of genes associated with virus receptor activity (GO category) revealed lower ACE2 gene expression in S. epidermidis-inoculated NHNE cells (0.51-fold decrease) relative to the control (Fig. 2c). We also analyzed significant gene expression associated with serine-type peptidase activity (GO category) and found that TMPRSS2 expression was decreased in S. epidermidis-inoculated NHNE cells (0.59-fold decrease) relative to the control (Fig. 2d). Interestingly, of the scatter plot data of genes associated with GO category, serine-type peptidase inhibitor activity showed that both SERPINE1 (17.2-fold increase) and SERPINE2 (40.8-fold increase) expression was significantly elevated in $S$. epidermidis-inoculated NHNE cells (Fig. 2e).

Next, we analyzed the significant change of ACE2, TMPRSS2, SERPINE1, and SERPINE2 expression in NHNE cell subsets of basal cells, secretory-like cells, undefined intermediate cells, suprabasal cells, and multiciliated cells in the presence or absence of $S$. epidermidis. Heatmap data depicting genes classified into virus receptor activity revealed that decrease in ACE2 transcription in response to $S$. epidermidis inoculation was most pronounced in basal cells and secretory-like NHNE cells. A decrease of ACE2 gene expression was observed in suprabasal and undefined intermediate cells of $S$. epidermidis-inoculated NHNE cells. ACE2 gene expression was not significantly altered in multiciliated cells (Fig. 2f).

Based on dot plot data, normalized TMRPSS2 expression was significantly higher in multiciliated cultured NHNE cells, and a larger proportion of SERPINE1 and SERPINE2 gene expression was found in basal NHNE cells (Fig. 2g). The heatmap of serine-type peptidase transcript activity also showed that TMPRSS2 transcription was significantly reduced in multiciliated $S$. epidermidis-inoculated NHNE cells (Fig. $2 \mathrm{~h}$ ). In contrast, a significant increase of serine-type peptidase inhibitor SERPINE1 and SERPINE2 transcripts was determined in all NHNE basal, secretory-like, suprabasal, and undefined intermediate cell subsets with $S$. epidermidis inoculation. The baseline transcript levels of SERPINE1 and SERPINE2 were minimal in multiciliated cells, but the expression of both was highly induced after $S$. epidermidis inoculation (Additional file 1: Figure S3). Based on these findings, we suggest that human nasal commensal $S$. epidermidis reduced gene expression associated with host entry of SARS-CoV-2, including ACE2 and TMPRSS2, depending on NHNE cell subset. Contrary to these findings, S. epidermidis inoculation enhanced the gene expression of serine-type protease inhibitors SERPINE1 and SERPINE2, which might be involved in reduction of serine protease activity, including TMPRSS2, in NHNE cells.

To better determine the influence of $S$. epidermidis on SARS-CoV-2 host entry factors in the nasal epithelium, NHNE cells from five healthy subjects were inoculated with human nasal $S$. epidermidis at an MOI of 0.25, and transcriptional changes of ACE2, TMPRSS2, SERPINE1, and SERPINE2 were evaluated. S. epidermidis mRNA level in the cell lysate and the colony count of $S$. epidermidis in the supernatant 
were assessed until 1-day post infection (dpi). Real-time PCR data revealed that the mean mRNA level of S. epidermidis femA increased significantly starting from $8 \mathrm{~h}$ post infection $\left(0.8 \times 10^{9}\right)$, and that the highest levels were observed at $1 \mathrm{dpi}\left(4.2^{\prime} 10^{9} ; \mathrm{Fig}\right.$. 3a). The mean colony forming unit (CFU) of $S$. epidermidis was significantly increased in the supernatant of $S$. epidermidis-inoculated NHNE cells until 1 day $\left(2.4 \times 10^{4} \mathrm{CFU} / \mathrm{ml}\right)$ after $S$. epidermidis inoculation (Fig. 3b). Subsequently, we tested whether $S$. epidermidis-inoculated NHNE cells exhibited the decrease of ACE2 and TMPRSS2 as shown in scRNA-seq data. Real-time PCR and immunohistochemistry results showed that ACE2 mRNA and protein levels were reduced significantly at 1 day after $S$. epidermidis inoculation (Fig. 3c, 3d). In addition, TMRPSS2 mRNA level decreased significantly in the cell lysates of $S$. epidermidis-inoculated NHNE cells until 1 day after inoculation. A gradual increase of SERPINE1 and SERPINE2 gene expression was seen in NHNE cells in response to $S$. epidermidis, with the highest expression observed at $24 \mathrm{~h}$ after inoculation (Fig. $3 \mathrm{e}$ ).

Considering the in vitro effect of the nasal commensal S. epidermidis on entry factors of SARS-CoV-2 in the nasal epithelium, we investigated the relationship between $S$. epidermidis abundance and mRNA levels of ACE2 and TMPRSS2 in human nasal mucosa. Nasal mucus and middle turbinate mucosa of 20 healthy subjects was collected, and the number of $S$. epidermidis CFUs from nasal mucus and ACE2 or TMPRSS2 mRNA levels from the human nasal mucosa were compared. Interestingly, S. epidermidis CFUs from healthy human nasal mucus was inversely correlated with ACE2 (Spearman $r=-0.7469$ ) and TMPRSS2 (Spearman $r=-0.6581$ ) mRNA levels in the nasal mucosa (Fig. $3 \mathrm{f}, 3 \mathrm{~g}$ ). These data indicate that subjects who have more number of $S$. epidermidis in their nasal mucus show relatively lower levels of ACE2 and TMPRSS2 gene expression, and that subjects with decreased number of $S$. epidermidis in the nasal mucus have higher transient expression of ACE2 and TMPRSS2.

\section{Discussions}

Altogether, our findings indicate that the most abundant human nasal commensal, S. epidermidis, restricts host entry of SARS-CoV-2 into the nasal epithelium through reduction of host virus receptors and a principal host protease that are necessary for cellular transmission. In addition, $S$. epidermidis can increase expression of the serine-type protease inhibitors SERPINE1 and SERPINE2 in nasal epithelial cells.

Host protection against viral infections can be conferred by the nasal microbiome via a specialized immune mechanism and recent work has highlighted $S$. epidermidis is capable of combating invasion by respiratory viruses [13]. Growing evidence shows that the entry factors for SARS-CoV-2, including ACE2 and TMPRSS2, are dominantly found in the nasal epithelium, and nasal epithelial cells have been determined as a potential cellular target of SARS-CoV-2 infection [17-20]. Thus, we characterized the contribution of the nasal commensal $S$. epidermidis to the defense mechanisms against SARS-CoV-2 infection, which mainly targets nasal epithelial cells. 
Our scRNA-seq findings indicate that primary nasal epithelial cells support entry of SARS-CoV-2 leading to spread to the respiratory tract, and the human nasal commensal $S$. epidermidis downregulated cellular entry factors in the nasal epithelium. Both ACE2 and TMPRSS2 transcription in the nasal epithelium was significantly reduced after inoculation with $S$. epidermidis in suprabasal, secretory-like, and multiciliated NHNE cells. This result is underscored by the inverse correlation between ACE2 and TMPRSS2 of the nasal mucosa and $S$. epidermidis colony number in human nasal mucus. Thus, a greater abundance of $S$. epidermidis in the nasal mucus results in lower ACE2 and TMPRSS2 expression in the nasal mucosa of healthy subjects.

\section{Conclusions}

The present study suggests that the nasal commensal S. epidermidis-regulated transcription of ACE2, TMPRSS2, SERPINE1, and SERPINE2 in nasal epithelium, even in a cellular environment free from SARSCoV-2 infection and S. epidermidis, can restrict cellular entry factors of SARS-CoV-2 in nasal epithelial cells to impede SARS-CoV-2 invasion into the human respiratory tract. Our work highlights the importance of host-bacterial commensalism in shaping the cellular environment of the nasal epithelium, resulting in decreased SARS-CoV-2 invasion into epithelial cells through modulation of host entry factors.

\section{Methods}

\section{Subjects and sample collection}

The $1 \times 1-\mathrm{cm}$-sized nasal mucosal tissue samples from the middle turbinate of the subjects $(\mathrm{N}=4)$ who underwent septoplasty under general anesthesia in the Department of Otorhinolaryngology Seoul National University Hospital (Seoul, Korea) were obtained for real-time PCR. Also, 1×1-cm-sized human lung parenchymal tissues of the subjects $(\mathrm{N}=4)$ were obtained from the subjects referred to the Department of Thoracic and Cardiovascular Surgery Seoul National University Hospital, primarily for pneumonectomy.

\section{Nasal mucus S. epidermidis characterization}

Mucus from the middle turbinate of healthy volunteers was collected individually using sterile 3M Quick swabs (3M Microbiology Products, St. Paul, MN, USA) from four subjects using a rigid 0-degree endoscope in an operating room. The swabs with mucus were fixed in a fixative solution and transported immediately to the laboratory for identification and subsequent microbial analysis. For bacterial colony isolation, the mucus was plated on Lysogeny Broth (LB) plates. After two days of incubation, bacterial colonies were obtained from the LB plates, and the species of each colony was identified using GS-FLX 454 pyrosequencing and $16 \mathrm{~S}$ rRNA gene amplification. ${ }^{13}$ Four S. epidermidis strains were isolated from four individuals. 


\section{Cell culture}

Normal human nasal epithelial (NHNE) cells were cultured as described previously [21]. Briefly, passage-2 NHNE cells $\left(1 \times 10^{5}\right.$ cells/culture) were seeded in $0.25 \mathrm{ml}$ of culture medium on Transwell-Clear culture inserts (24.5 mm, with a 0.45-mm pore size; Costar Co., Cambridge, MA, USA). Cells were cultured in a 1:1 mixture of basal epithelial growth medium and DMEM containing previously described supplements. Cultures were grown while submerged for the first 9 days. The culture medium was changed on Day 1 and every other day thereafter. An air-liquid interface (ALI) was created on Day 9 by removing the apical medium and feeding the cultures from the basal compartment only. The culture medium was changed daily after establishment of the ALI. The antifungal agent fungizone (1 ml / $1000 \mathrm{ml}$ media) (Life Technologies, Grand Island, NY, USA) was added after filtering the media. All experiments described here used cultured nasal epithelial cells at 14 days after ALI.

\section{Single-cell RNA sequencing (scRNA-seq)}

Library construction was performed using 10X Chromium Single Cell 3' reagent kits v3.1. Samples were sequenced using the Illumina NovaSeq 6000 platform, and preliminary sequencing results were converted to FASTQ files using the Cell Ranger pipeline. We followed the 10x Genomics standard sequence protocol by trimming the barcode and unique molecular identifier (UMI) end to $26 \mathrm{bp}$ and the mRNA end to $98 \mathrm{bp}$. Then, the FASTQ files were aligned to the human reference genome (GRCh38). Subsequently, we applied Cell Ranger for preliminary data analysis and generated a file that contained a barcode table, a gene table, and a gene expression matrix. We used the WinSeurat v2.1 (Ebiogen Inc., Seoul, Korea) based on Seurat version 3 for QC, analysis, and exploration of single-cell RNA-seq data [22, 23]. Data mining and graphic visualization were performed using ExDEGA (Ebiogen Inc., Seoul, Korea).

\section{Real-time PCR}

NHNE cells were infected with $S$. epidermidis for 4,8 , or $24 \mathrm{~h}$, and total RNA was isolated using TRIzol (Life Technology, Seoul, Korea). cDNA was synthesized from $3 \mu \mathrm{g}$ of RNA with random hexamer primers and Moloney murine leukemia virus reverse transcriptase (Perkin Elmer Life Sciences, Waltham, MA, USA and Roche Applied Science, Indianapolis, IN, USA). Amplification was performed using the TaqMan Universal PCR master mix (PE Biosystems, Foster City, CA, USA) according to the manufacturer's protocol. Briefly, $12 \mu \mathrm{l}$ amplification reactions contained $2 \mu \mathrm{l}$ of CDNA (reverse transcription mixture), oligonucleotide primers (final concentration of $800 \mathrm{nM}$ ), and TaqMan hybridization probe (200 nM). Realtime PCR probes were labeled at the $5^{\prime}$ end with carboxyfluorescein (FAM) and at the $3^{\prime}$ end with the quencher 5-carboxytetramethylrhodamine (5-TAMRA). To quantify cellular viral level and host gene 
expression, cellular RNA was used to generate cDNA. Primers for femA, SERPINE1, SERPINE2, TMPRSS2, and ACE2 were purchased from Applied Biosystems (Foster City, CA, USA). Real-time PCR was performed using the PE Biosystems ABI PRISM ${ }^{\circledR} 7700$ Sequence Detection System. Thermocycler parameters were as follows: $50^{\circ} \mathrm{C}$ for $2 \mathrm{~min}, 95^{\circ} \mathrm{C}$ for $10 \mathrm{~min}$, and 40 cycles of $95^{\circ} \mathrm{C}$ for $15 \mathrm{~s}$ and $60^{\circ} \mathrm{C}$ for $1 \mathrm{~min}$. Target mRNA levels were quantified using target-specific primer and probe sets for femA, SERPINE1, SERPINE2, TMPRSS2, and ACE2. All PCR assays were quantitative and utilized plasmids containing the target gene sequences as standards. All reactions were performed in triplicate, and all real-time PCR data were normalized to the level of glyceraldehyde phosphate dehydrogenase (GAPDH, $1^{\prime} 10^{6}$ copies) to correct for variations between samples.

\section{Western blot analysis}

Protein level of ACE2 was assessed using western blot analysis, and the monoclonal antibody of ACE2 was purchased from Cell Signaling Technology (Beverly, MA, USA). The NHNE cells and were lysed with $2 \mathrm{X}$ lysis buffer (250 mM Tris-Cl (pH6.5), 2\% SDS, 4\% $\beta$-mercaptoethanol, 0.02\% bromophenol blue, and $10 \%$ glycerol). Cell lysate (30 $\mu \mathrm{g}$ of protein) was electrophoresed in $10 \%$ SDS gels and transferred to polyvinylidene difluoride membranes in Tris-buffered saline (TBS; $50 \mathrm{mM}$ Tris- $\mathrm{Cl}(\mathrm{pH} 7.5), 150 \mathrm{mM} \mathrm{NaCl}$ ) for $1 \mathrm{~h}$ at room temperature. The membrane was incubated overnight with primary antibody (1:500) in Tween-Tris buffered saline (TTBS; 0.5\% Tween-20 in TBS). After washing with TTBS, the blot was incubated for $1 \mathrm{~h}$ at room temperature with secondary anti-rabbit antibody (1:1000, Cell Signaling, Beverly, MA, USA) in TTBS and was visualized using an ECL system (Amersham, Little Chalfont, UK).

\section{Immunohistochemistry}

Human nasal mucosa was obtained from the middle turbinate of healthy subjects who had nasal surgery due to nasal obstruction, and paraffin block slides were prepared for immunostaining. Immunohistochemical analysis was performed using ACE2 antibody (1:200, Cell Signaling Technology, MA, USA) to determine protein level in the nasal mucosa. Briefly, 5 - $\mu \mathrm{m}$ sections were fixed in acetone for 10 min at room temperature (RT). Non-specific protein staining was blocked with goat serum. Slides were treated with $0.5 \%$ hydrogen peroxidase to eliminate endogenous peroxidase for $10 \mathrm{~min}$ at RT and incubated with primary antibody overnight at RT. After washing with Tris-buffered saline (TBS, pH 7.5), slides were incubated with horseradish peroxidase-conjugated secondary antibody (Thermo, Asheville, NC, USA) for 30 min at RT. Chromogen (3-amino-9-ethylcarbazole) was applied for visualization. Glass cover slides were mounted and examined with optical microscopy and ACE2 protein was detected with DAB (3,3'-diaminobenzidine) chromogen staining. The same procedures were performed using nonimmunized mouse IgG (purified IgG, Sigma) instead of primary antibody as a negative control. 


\section{Statistical analyses}

For in vitro study, at least three independent experiments were performed with cultured cells from each donor, and the results are presented as the mean value \pm standard deviation (SD) of triplicate cultures. Differences between treatment groups were evaluated by analysis of variance (ANOVA) with a post hoc test. We present the in vivo results of real-time PCR, plaque assays, and ELISA as mean value \pm SD from five individual mice. Statistical analyses were performed using GraphPad Prism software (version 5; GraphPad Software, La Jolla, CA, USA). A p-value $<0.05$ was considered statistically significant.

\section{Declarations}

\section{Additional files}

Additional file 1: Figure S1-S3. This file contains the supplementary figures.

\section{Acknowledgements and funding}

This work was supported by the Basic Science Research Program through the National Research Foundation of Korea funded by the Ministry of Education (2016R1D1A1B01014116 and 2019M3C9A6091945 to HJK). This research was also supported by a grant from the Korea Health Technology R\&D Project through the Korea Health Industry Development Institute (KHIDI), funded by the Ministry of Health \& Welfare of the Republic of Korea (HI20C0546 to HJK).

\section{Availability of data and materials}

Not applicable

JYJ and HJK conceived the study and designed the experiments. JYJ and AJ carried out the study including sample collection and sample preparation. JW, CHG, HS, YJJ, and SK performed additional work, design, and data analysis. AJ and HJK drafted the manuscript.

\section{Competing interests}

The authors declare that the research was conducted in the absence of any commercial or financial relationships that could be construed as potential conflicts of interest. 


\section{Ethics approval and consent to participate}

Participation was voluntary, with written informed consent obtained from all subjects. This study was approved by the Institutional Review Board (IRB) of the Seoul National University College of Medicine (no. 1709-049-883).

\section{References}

1. Zhou, P. et al. Addendum: A pneumonia outbreak associated with a new coronavirus of probable bat origin. Nature 588, E6 (2020).

2. Paules, C.I., Marston, H.D., Fauci, A.S. Coronavirus Infections-More Than Just the Common Cold. JAMA 323, 707-708 (2020).

3. Wang, M.Y. et al. SARS-CoV-2: Structure, Biology, and Structure-Based Therapeutics Development. Front. Cell. Infect. Microbiol. 10, 587269 (2020).

4. Lederer, K. et al. SARS-CoV-2 mRNA Vaccines Foster Potent Antigen-Specific Germinal Center Responses Associated with Neutralizing Antibody Generation. Immunity 53, 1281-1295 (2020).

5. Laporte, M., Naesens, L. Airway proteases: an emerging drug target for influenza and other respiratory virus infections. Curr. Opin. Virol. 24, 16-24 (2017).

6. Bertram, S. et al. Novel insights into proteolytic cleavage of influenza virus hemagglutinin. Rev. Med. Virol. 20, 298-310 (2010).

7. Dittmann $M$, et al. A serpin shapes the extracellular environment to prevent influenza $A$ virus maturation. Cell 160, 631-643 (2015).

8. Sungnak, W. et al. SARS-CoV-2 entry factors are highly expressed in nasal epithelial cells together with innate immune genes. Nat. Med. 26, 681-687 (2020).

9. Blanco-Melo, D. et al. Imbalanced Host Response to SARS-CoV-2 Drives Development of COVID-19. Cell 2020 181, 1036-1045 (2020).

10. Onabajo, O.O. et al. Interferons and viruses induce a novel truncated ACE2 isoform and not the fulllength SARS-CoV-2 receptor. Nat. Genet. 52, 1283-1293 (2020).

11. Stanifer, M.L. et al. Critical Role of Type III Interferon in Controlling SARS-CoV-2 Infection in Human Intestinal Epithelial Cells. Cell. Rep. 32, 107863 (2020).

12. Segal, L.N., Rom, W.N., Weiden, M.D. Lung microbiome for clinicians. New discoveries about bugs in healthy and diseased lungs. Ann. Am. Thorac. Soc. 11, 108-116 (2014).

13. Kim, H.J. et al. Nasal commensal Staphylococcus epidermidis enhances interferon- $\lambda$-dependent immunity against influenza virus. Microbiome 7, 802019 (2019). 
14. Planet, P.J. et al. Lambda Interferon Restructures the Nasal Microbiome and Increases Susceptibility to Staphylococcus aureus Superinfection. MBio 7, e01939-15 (2016).

15. Jeon, Y.J. et al. The influence of interferon-lambda on restricting Middle East Respiratory Syndrome Coronavirus replication in the respiratory epithelium. Antiviral. Res. 180, 104860 (2020).

16. Broszeit, F. et al. N-Glycolylneuraminic Acid as a Receptor for Influenza A Viruses. Cell. Rep. 27, 32843294 (2019).

17. Gallo O, Locatello LG, Mazzoni A, Novelli L, Annunziato F. The central role of the nasal microenvironment in the transmission, modulation, and clinical progression of SARS-CoV2 infection. Mucosal Immunol. 2020 Nov 26:1-12.

18. 20. Kim, S. et al. The Superiority of IFN- $\lambda$ as a Therapeutic Candidate to Control Acute Influenza Viral Lung Infection. Am. J. Respir. Cell. Mol. Biol. 56, 202-212 (2017).

19. Won, J., Gil, C.H., Jo, A., Kim, H.J. Inhaled delivery of Interferon-lambda restricts epithelial-derived Th2 inflammation in allergic asthma. Cytokine. 119, 32-36 (2019).

20. An, S. et al. Initial Influenza Virus Replication Can Be Limited in Allergic Asthma Through Rapid Induction of Type III Interferons in Respiratory Epithelium. Front. Immunol. 9, 986 (2018).

21. Park, D.Y. et al. Alternative Method for Primary Nasal Epithelial Cell Culture Using Intranasal Brushing and Feasibility for the Study of Epithelial Functions in Allergic Rhinitis. Allergy. Asthma. Immunol.

Res. 8, 69-78 (2016).

22. Butler, A. et al. Integrating single-cell transcriptomic data across different conditions, technologies, and species. Nat. Biotechnol. 36, 411-420 (2018).

23. Stuart, T. et al. Comprehensive Integration of Single-Cell Data. Cell 177, 1888-1902 (2019).

\section{Figures}
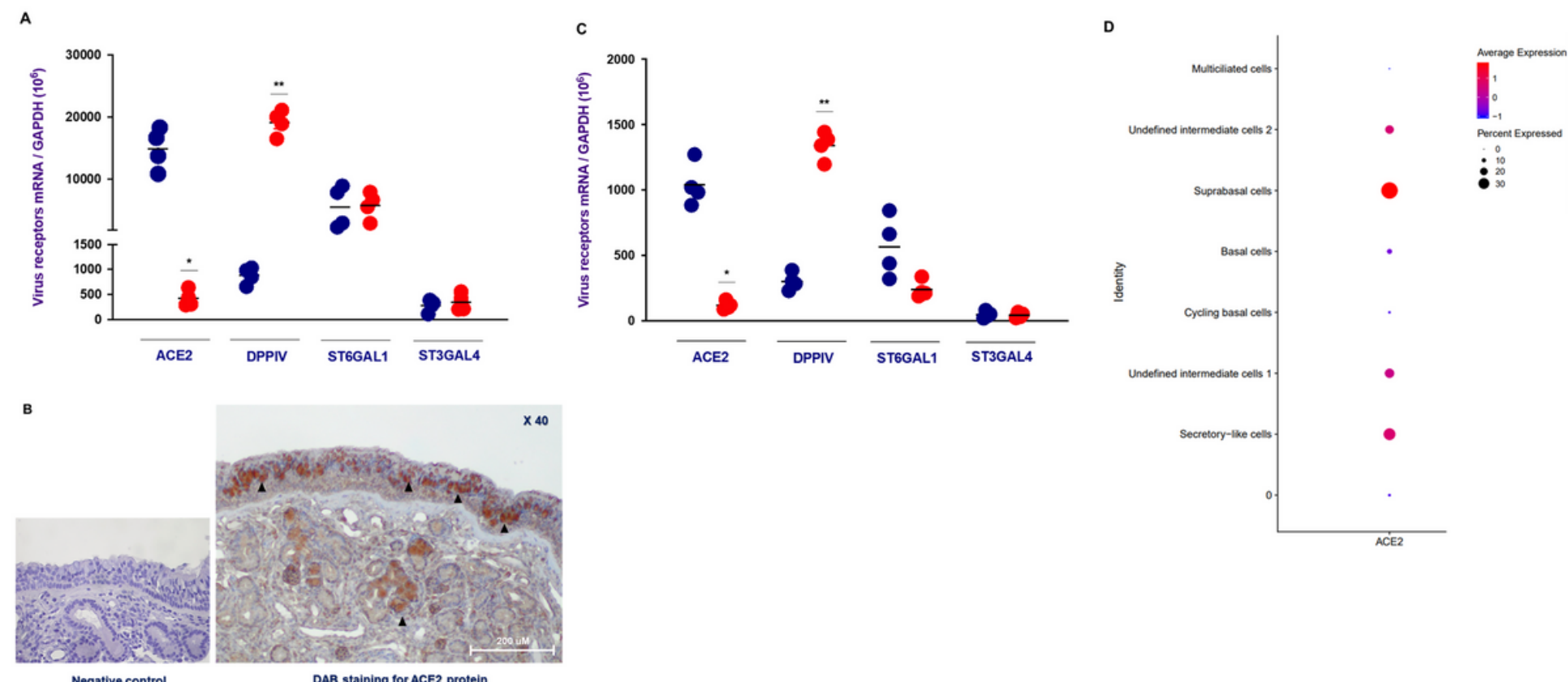


\section{Figure 1}

Expression of ACE2 in human nasal mucosa and lung tissue. a. RNA expression of ACE2, DPPIV, ST6GAL1, and ST3GAL4 was evaluated using nasal mucosal tissues (blue circles, $N=4$ ) and lung parenchymal tissues (red circles, $\mathrm{N}=4$ ) of healthy subjects. Compared to other virus receptors, ACE2 RNA expression was relatively higher in the human nasal mucosa. b. Immunohistochemistry (IHC) for ACE2 protein was performed using human nasal mucosa of middle turbinate and representative images of ACE2 IHC staining revealed that positive staining of DAB (3,3'-diaminobenzidine) (black arrows) was dominant in the nasal epithelium (original magnification $\times 40$ ). c. RNA expression of ACE2, DPPIV, ST6GAL1, and ST3GAL4 was evaluated using cultured normal human nasal epithelial (NHNE) cells (blue circles, $\mathrm{N}=4$ ) and normal human bronchial epithelial (NHBE) cells (red circles, $\mathrm{N}=4$ ). d. Expression of ACE2 from single-cell RNA sequencing (scRNA-seq) of NHNE cells. Dot size represents the proportion of ACE2 RNA expression within the respective cell type expressing the gene, and dot color represents the average ACE2 RNA expression level in the particular cell type. The results of real-time PCR are presented as the mean of human tissue or cultured epithelial cells from four subjects, and the $\mathrm{IHC}$ imaging is representative of four independent experiments. 


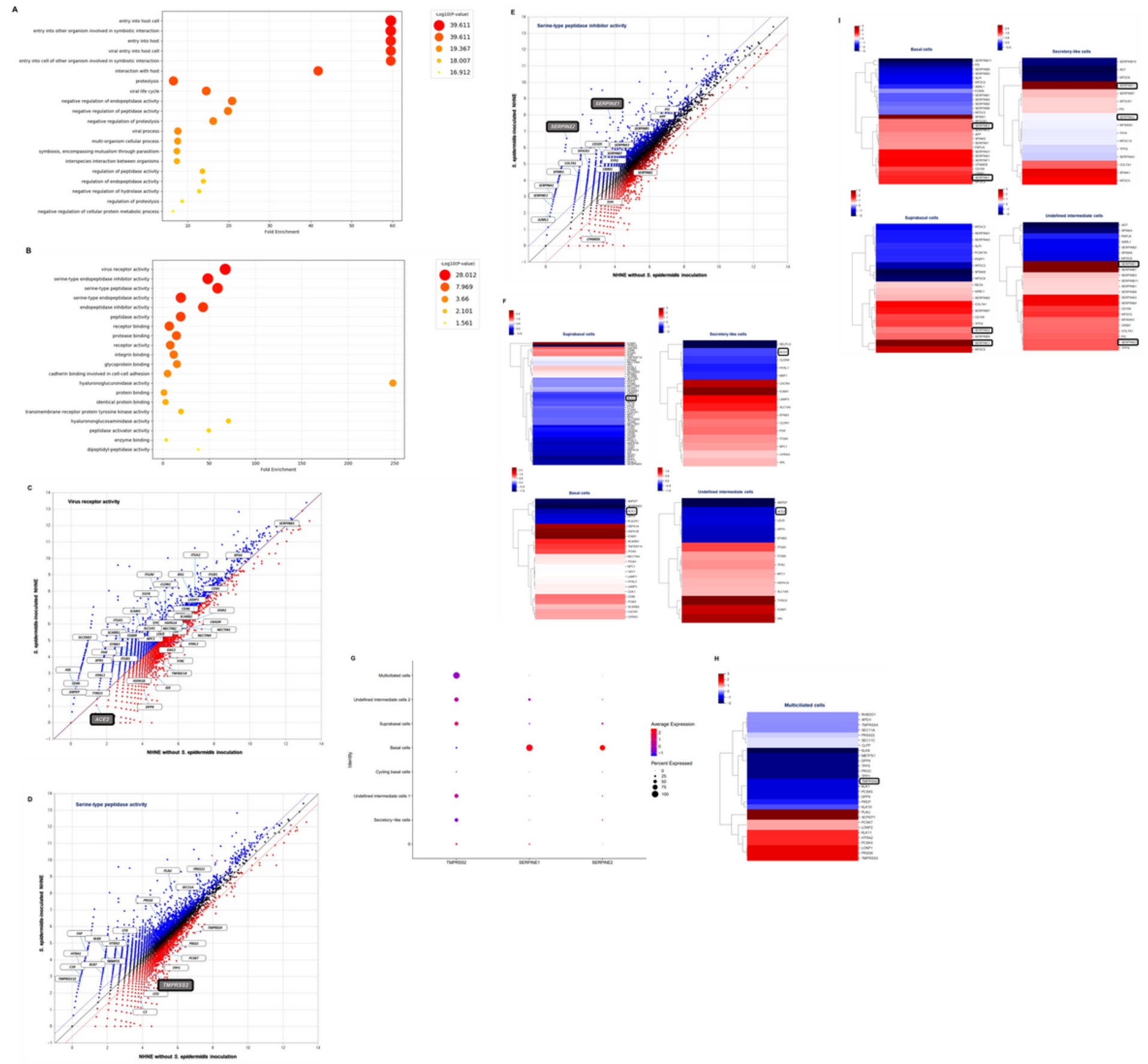

Figure 2

Host transcriptional response to S. epidermidis in NHNE cells. NHNE cells from four healthy volunteers were inoculated with human nasal S. epidermidis at an $\mathrm{MOI}$ of 0.25 , and total RNA was harvested after $24 \mathrm{~h}$. Dot plot visualization of enriched Gene Ontology (GO) terms related with a. "virus receptor activity" and b. "serine-type peptidase activity" in S. epidermidis-inoculated NHNE cells. Scatter plots indicating enriched genes related with c. virus receptor activity, d. serine-type peptidase, and e. serine-type peptidase activity in S. epidermidis-inoculated NHNE cells. f. Heatmap depicting the expression levels of genes related with virus receptor activity differentially expressed in S. epidermidis-inoculated NHNE cells depending on cellular subset. g. Dot size represents the proportion of TMPRSS2, SERPINE1, and 
SERPINE2 mRNA expression in respective cell types expressing the gene, and dot color represents the average RNA expression levels of the particular cell type. Heatmap depicting the expression levels of genes related with $\mathrm{h}$. serine-type peptidase activity and i. serine-type peptidase inhibitor activity differentially expressed in S. epidermidis-inoculated NHNE cells depending on cellular subset.

A

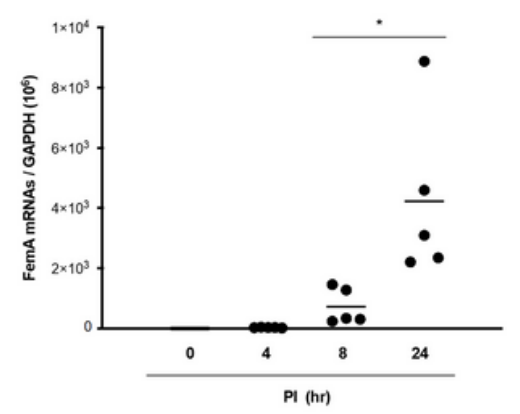

B
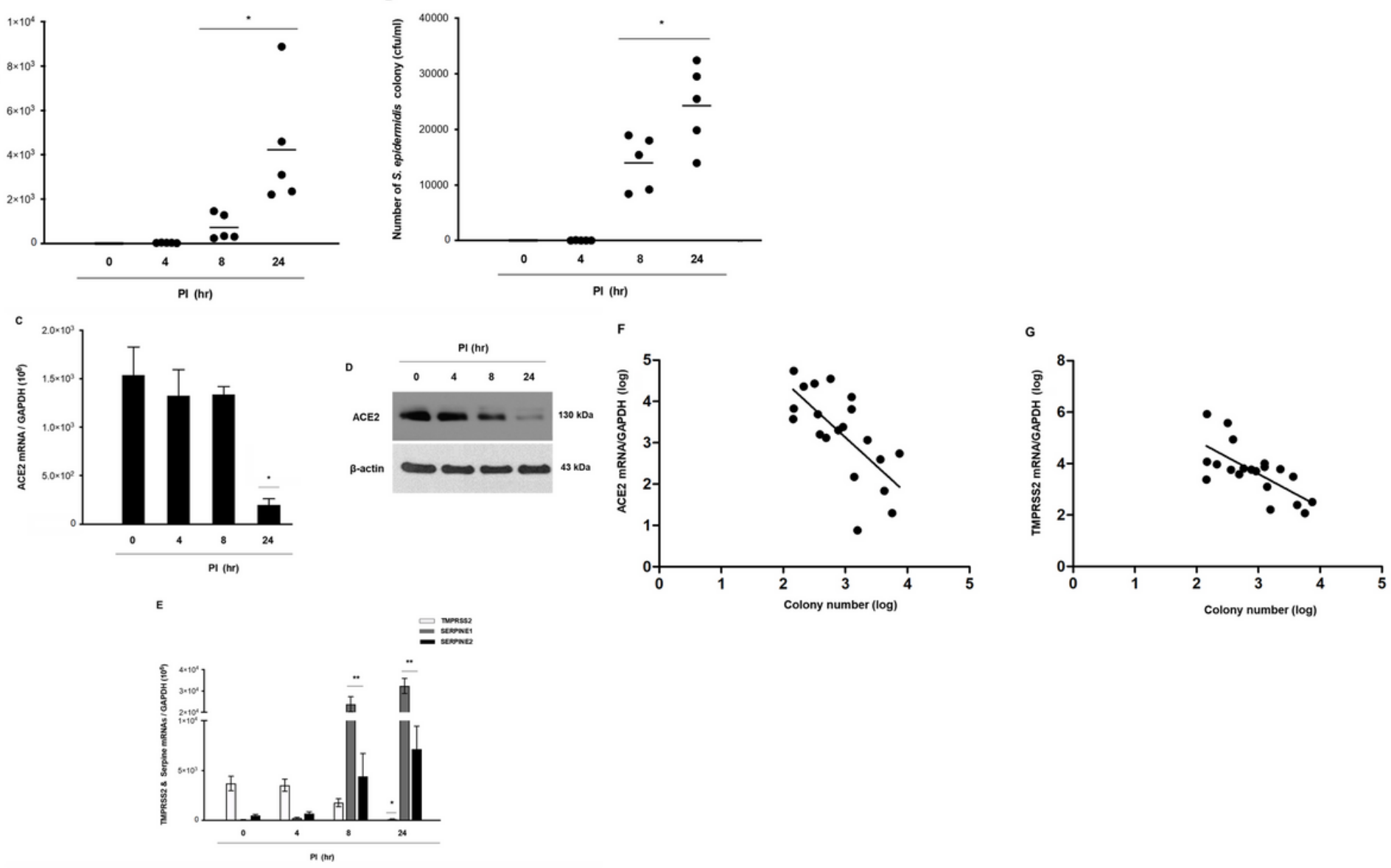

Figure 3

S. epidermidis promotes reduction of ACE2 and TMPRSS2 expression in the nasal epithelium and increases SERPINE1 and SERPINE2 transcription. NHNE cells from five healthy volunteers were inoculated with human nasal S. epidermidis at an $\mathrm{MOI}$ of 0.25 . a. femA mRNA level, normalized to cellular GAPDH transcript level, was monitored by real-time PCR at 4, 8, and 24 h. b. S. epidermidis CFUs were determined over the course of $24 \mathrm{~h}$ in the supernatant of $\mathrm{S}$. epidermidis-inoculated NHNE cells. c. ACE2 mRNA level, normalized to cellular GAPDH transcript level, was determined by real-time PCR until 24 h. d. The intracellular protein level of ACE2 was measured in the cell lysate of S. epidermidis-inoculated NHNE cells until $24 \mathrm{~h}$ using western blot analysis. e. TMPRSS2, SERPINE1, and SERPINE2 mRNA levels, normalized to cellular GAPDH transcript level, were determined by real-time PCR at 4, 8, and $24 \mathrm{~h}$. The mRNA levels of $f$. ACE2 and $g$. TMPRSS2 in the nasal mucosa from the middle turbinate of healthy volunteers $(n=20)$, as measured by real-time PCR, were correlated with colony-forming units (CFUs) of S. epidermidis isolated from the mucus of the middle turbinate collected from the same subjects. The correlation was determined using Spearman's correlation analysis (ACE2 (Spearman $r=-0.7469$, 
$p=0.0002)$ and TMPRSS2 (Spearman $r=-0.6581, p=0.0016)$. The western blot is representative of five independent experiments and real-time PCR results are presented as mean \pm SD from five independent experiments. ${ }^{*} p<0.05$ compared with NHNE cells without $S$. epidermidis inoculation.

\section{Supplementary Files}

This is a list of supplementary files associated with this preprint. Click to download.

- Additionalfile1.doc 